\title{
Index of names, main concepts and texts
}

Confucius' name was not included due to its too many occurrences

Aelred of Riveault 198

- De spirituali amicitia 198

Aeneas 102, 169

Alexander the Great 15

Ambrose of Milan 25

Aristotle $13,19,40-41,75,92,107,123$, 124, 191, 194

- Politics 40

Augustine of Hippo 25

Bachtin, Michail 75 f., 92

BNF Par. Lat.1687 115, 117

BNF Par. Lat. 6277 110, 112-113, 116-121

Buddhism 19, 51, 85, 179

buxiao (不孝) 164, 168

Caesar, C. Iulius $13,15 \mathrm{f} ., 18,20,22,24 \mathrm{f}$., 192

Cain 150

Capito, T. Roscius 155

Carneades 194

Catiline (L. Sergius Catilina) 22, 192

Ceng Can (see Zeng Shen/Zengzi)

Cheung Dasan (다산 정약용) 123

Cheung, William 2, 74, 78-80, 82-84, 86-90, 92-96, 98f., 102-104, 106

chi (恥/耻) 83, 89, 178

chi (智) (see zhi)

China 1, 3, 7 f., 10, 13, 21, 23, 25, 29 f., 36, 40 f., $60,63,74 f ., 78,84,94,107-110$, 123, 148, 171-182, $190 \mathrm{f} ., 195 \mathrm{f}$.

Chineseness 172

Chrysogonus $155 \mathrm{f}$.

Chu (楚) 35, 60, 101, 109-111, 115, 117 120, 123, 172, 181

Chun Qiu/Chunqiu/ Spring and Autumn Annals (春秋) 8, 10, 21, 73

Cicero, M. Tullius $1-4,6-25,29,48,74 \mathrm{f}$., $79,84,88,90,92,106,109-114,116-$
118, 124, 129-139, $144 \mathrm{f} ., 155-162$,

164, 166, 168, 189-195, 197-199

- De amicitia 198

- De finibus bonorum et malorum 161, 194

- De inventione $159 \mathrm{f}$.

- De natura deorum 116, 161, 194

- De officiis 10-19, 79, 88, 111, 113f., 134-136, 144

- De oratore 19, $132 \mathrm{f}$.

- De senectute 84

- Epistulae ad familiares 18, 20

- In Verrem 134

- Partitiones oratoriae $159 \mathrm{f}$

- Pro Archia 134

- Pro Sexto Roscio Amerino 3, 155-157, $162 \mathrm{f}$.

- Topica 161

Confucianism (儒家) 9, 11, 15, 19, 24, 34, $40,60,69,73,85,91,96,105,107$, $142,144,146,162,164-165,168,173-$ $176,179,182,195$

- Five Books (Five Qing [五經/五经]) 73

- Book of Changes (易經/易经) 11, 53, 73, $152 \mathrm{f}$.

- Book of History or Book of Documents (see Shujing) 8, 10, 21, 73

- Book of Rites (see Liji)

- Book of Songs 7-9, 12, 21, 73

- Four Books (Four Shu [四書/四书]) 73

- Analects or Dialogues (see Lunyu)

- The Book of Mencius (孟子) 73, 109

- The Doctrine of the Mean (see Zhongyong)

- The Great Learning (see Zeng Shen/Zengzi

- Spring and Autumn Annals (see Chun Qiu)

Confucius Sinarum Philosophus sive Scientia Sinensis 3, 73, 74, 109 
Couplet, Philippe 2, 73f., 77-80, 82-84, $86-90,92-99,101-106,109$ f., $115-$ 117

Couvreur, Seraphin $74,78-80,82-84$, 86-90, 92-96, 98f., 101-104, 106

Crassus, M. Licinius 19, 22, $132 \mathrm{f}$.

Crown Prince Sohyeon (소현세자) 148

Cultural Revolution 107, 172-175

cursus honorum $190 \mathrm{f}$.

dao (道) $46,61,94-96,152,163,166$, 182, 196

Daoism (道家) 10, 19, 43-45, 51-52

Daxue/ Ta-Hio / Magna Scientia (大學/大学) (see Zeng Shen/Zengzi) 76, 109-110, 121, 149, 153

decemviri 155, 159

Diogenes 45

Education $8,18,20-22,25,37,39$ f., 62, $76 f ., 130-133,136 f ., 164-166,173$, 179, $189 \mathrm{f} ., 193$

Empedocles 49

Epictetus 51, 53, 116

Epicurus /Epicurean/Epicureanism 43-45, $52-56,194,197,198$

- Fragments 44-45, 55

- Letter to Menoeceus 45

Equivocation 3, $129 \mathrm{f}$., 139

Erasmus of Rotterdam 131, 138

Erucius $155 \mathrm{f} ., 158 \mathrm{f}$.

Feng Guifen（冯桂芬） 172

Genesis 150

gin (仁) (see ren) 113, 116

God-model 195f., 198

Greece 8, 22, $191 \mathrm{f}$.

Han Fei Zi/Han Feizi (韓非子/韩非子) $34-35,41,60$

Hegel, Georg Wilhem Friedrich 114

Hêgemonikon 116

Hellenism, Hellenistic 124, 160, 191, 194

Heraclitus 49, 54

Herdtrich, Christian 73f., 109

Homer /Homeric 9, 90

29 ,
Hu Jintao (胡锦涛) 177, 180

Humanism 20

humanitas $3,10,14,16-20,105 \mathrm{f} ., 113 \mathrm{f}$., $129-139,155,158,160,167$

Hu Yaobang (胡耀邦) 175

Injo 148

Intorcetta, Prospero 1, 73f., 109-115, $117 \mathrm{f} ., 123 \mathrm{f}$.

iustitia $\quad 14,115,159,161,164,168$

Japan $1,107,163$

Jesuits $73,75,91,95,109$

Jesus $91,95,146,149-151,195$

jian (諫) 168

jian (儉/俭)181f., 185

Jie (然) 64

jing (敬) 149, 163, 165, 168

Jixia (稷下) 60

junzi (君子) 42, 61, 65, 77, 79, 81ff., 91, 95, 100-101, 112, 142, 145, 152, 182

Kant, Immanuel $\quad 32,122 \mathrm{f}$.

- Die Religion innerhalb der Grenzen der bloßen Vernunft 123

kien (謙) 114

Kong Ji (孔伋) 73

Korea 1, 3, 107, 147-149, 151f., $163 \mathrm{f}$.

Lactantius, L. Caecilius Firmianus 20, 25, 110, 114, $117 \mathrm{f} ., 132,137$

Lanling (蘭陵) 60

Lao Peng (老彭) 99

Laozi (老子) 2, 10-12, 14, 44, 63, 191

Law 6, 19, 34-36, 39, 61, 89-91, 95, 113, $115,119,123,130,133,138,146,157 \mathrm{f}$. $177 \mathrm{f} ., 192,194$

li (理) $60,65,116 \mathrm{f}$.

li (禮/礼) 2, 12-18, 61, 66 f., 81, 104 f., 113, 167,

Liji (禮記/礼记 or 礼经) 17, 73

Li Shen (李紳/李绅) 181

Lisi (李斯) 60

Livy $8,90,161$

Louis XIV 3,109f.

Lucilius 161

Lucretius $45 \mathrm{f}$., 52 


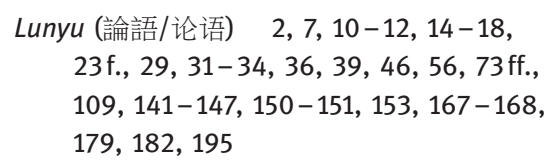

Lüshi Chunqiu (呂氏春秋) 142

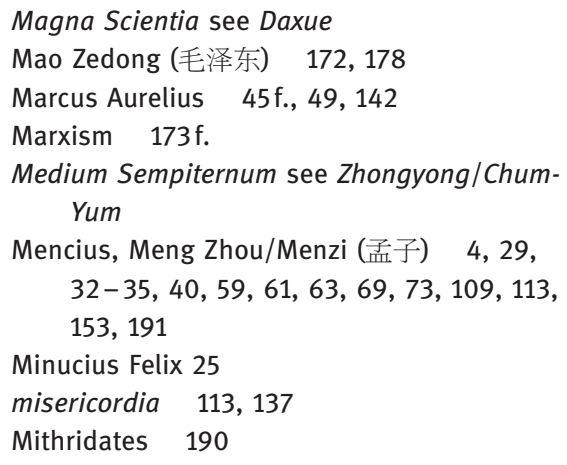

Magna Scientia see Daxue

Mao Zedong (毛泽东) 172, 178

Marcus Aurelius 45f., 49, 142

Marxism $173 \mathrm{f}$.

Medium Sempiternum see Zhongyong/ChumYum

Mencius, Meng Zhou/Menzi (孟子) 4, 29, $32-35,40,59,61,63,69,73,109,113$, 153, 191

Minucius Felix 25

misericordia 113,137

Mithridates 190

Nam-in 148

New/neo Confucianism 13, 116, 123, 149 150, 173

Nietzsche, Friedrich 49, 57, $81 \mathrm{f}$.

Noël, François 109, 168

Operation Empty Plate $171,181,184$

Ovid (P. Ovidius Naso) 118, 124, $160 \mathrm{f}$.

Panaetius 12f., 197

parricidium 155f., 158

pietas $3,87,102-104,113,155-169$

Plato 2, 12f, 15, 29 f., 35f., 41, 48, 50, 54, 57, 75, 81f., 116, 168, 191, 194, 198

- Eutyphro 116

- Gorgias 81

- Leges 50, 194

- Phaedo 38, 54

- Respublica 29, 31, 33-36, 38-42, 194

- Sophista 50

- Timaeus 48, 50

Plutarch 1, 116

poena cullei 164

Pompey 22, 192

Posidonius 197

probabilitas 113

Proverbs 77 qianjing (虔敬/敬虔) 163

Qi (齊) 60, 168, 190-192

qi (氣/气) $45-47,50-51,66$

Qin (秦) 24, 60, 179, 182

Qinshihuang (秦始皇) 60

Quintilian 25

Ratiocinantium Sermones (see Lunyu)

religio 106, 110, 115, 122f., 160-162, 164, 166, 195

ren (仁)/renyi (仁義/仁义) 3, 10, 13-20, 32, 59, 63, 65, $85 \mathrm{ff} .$, 99, 103, 105, 116, 141, 143, 145, 147, 151, 165, 167, $197 \mathrm{ff}$.

Ricci, Matteo 1, 73, 75, 88, 96, 109 f., 151

Rome $7 f$., 12, 15, 22, 73, 109f., 131, 155, 166, 169, 190-192, 195

Roscius, P. Sextus the elder $155 \mathrm{f}$.

Roscius, P. Sextus the younger $155 \mathrm{f}$.

Rougemont, François 73f., 109

Ruggieri, Michele $\quad 2,73,77,79 \mathrm{f}$., 82-84, 86-90, 92-100, 102-106, 109

Sceptic, Scepticism 191, 194, 198

Seneca, L. Annaeus 1f., 92, 116, 146

Seonggyo yoji (see Yi Byeok)

Seventeen years 172

shen (神/神) 61, 91, 94, 165

Shijing (詩經/诗经) (see Confucianism, Book of Songs)

Shiji/Shi Ji (史記/史记) 8, 35, 60

shu (述) 98-99

Shujing (書經/书经) 10,73

Shun (舜) 11, 13, 17f., 34, 143, 150

Sima Qian/ Simaqian (司馬遷/司马迁) 8, $10,35,60$

- Records of the Grand Historian (see Shiji)

Sinocentrism 191

Socrates 12f., 33, 36-40, 54-55, 81, 98, 107, 116, 195

Songjian (宋鈃) 63

Spinoza, Baruch 47

Stoicism/Stoics 11f., 14, 43-47, 49-50, 55, 57, 92, 96, 124, $130 \mathrm{f}$., $145 \mathrm{f},, 167$, 191, 194, $197 \mathrm{f}$.

taizi (太極) 116

Thomas Aquinas 75 
tian (天) 34, 59, 91, 149, 197

- tianguan (天官) 64

- tianjun (天君) 64

- tianming (天命) 91-92, 97

- tianyang (天養) 59, 64

- tianzheng (天政) 59, 64

Tian'anmen (天安门) Square 174, 176, 178

Tullia 193

urbanitas 113

Vietnam 107

Virgil (P. Vergilius Maro) 75, 90, 102, 161

Wang Yangming (王陽明) 33

wanwu (萬物) 196, 198

Warring States (戰國/战国) 31, 34, 60, 165, 189-191, 193

wen (文) 59-61, 68-70, 87ff.

Westernization 176

Wolff, Christian 110, 118-122, 124

- German Metaphysics 121

- Psychologia Rationalis 119-122, 124

xiao (孝) 3, 14, 102, 155, 162-169, 182

Xiaojing (孝經/孝经) 164-168

Xi Jinping (习近平) 3, 171, 178-181

xin (信) 14, 59, 96 f., 99

xing'e (性惡) 62

xue (學/学) 76-81, 96, 181f.

Xunzi/Xunzi (荀子) $59 \mathrm{ff}$.

yi (義/义) 31, 66, 113, 163, 196

Yak-yong Dasan (see Cheung Dasan) 148

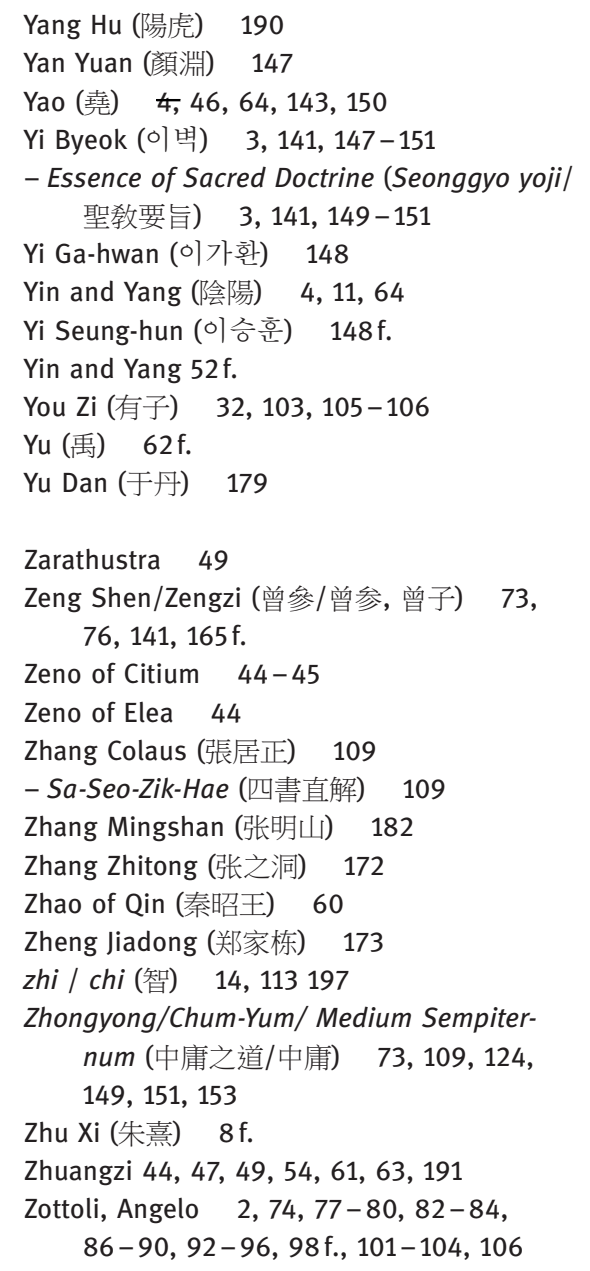

Yan Yuan (顏淵) 147

Yi Byeok (이벽) 3, 141, 147-151

Essence of Sacred Doctrine (Seonggyo yoji/ 3,141, 149-151

Yin and Yang (陰陽) 4, 11, 64

Yi Seung-hun (이승훈) $148 \mathrm{f}$.

Yin and Yang $52 \mathrm{f}$.

You Zi (有子) 32, 103, 105-106

Yu (禹) $62 \mathrm{f}$.

Yu Dan (于丹) 179

Zarathustra 49

eng Shen/Zengzi (曾參/曾参, 曾子) 73, $76,141,165 \mathrm{f}$

Zeno of Citium $\quad 44-45$

Zeno of Elea 44

Zhang Colaus (張居正) 109

- Sa-Seo-Zik-Hae (四書直解) 109

Zhang Mingshan (张明山) 182

Zhang Zhitong (张之洞) 172

Zhao of Qin (秦昭王) 60

Zheng Jiadong (郑家栋) 173

zhi / chi (智) 14, 113197 num (中庸之道/中庸) $73,109,124$, 149, 151, 153

Zhu Xi (朱喜) $8 \mathrm{f}$.

Zhuangzi 44, 47, 49, 54, 61, 63, 191

86-90, 92-96, 98f., 101-104, 106 